\title{
Éléments dont il faut tenir compte concernant les programmes d'immunisation obligatoire des enfants
}

\author{
Noni E MacDonald^, Eve Dubé2 ${ }^{1}$, Daniel Grandt ${ }^{3}$
}

\section{Résumé}

Les éclosions de maladies évitables par la vaccination se produisent même dans les pays qui ont un accès illimité et relativement équitable à ce service parce que les taux d'immunisation sont inférieurs aux niveaux requis pour une lutte efficace contre ces maladies. La réticence à se faire vacciner que l'on peut voir dans de nombreux pays, y compris au Canada, a mené à l'adoption ou au renforcement de la législation exigeant la vaccination des enfants dans certaines provinces. Bien que la vaccination obligatoire puisse sembler être la solution la plus simple à ce problème, son efficacité ne correspond pas toujours aux attentes. De nombreux pays, états, provinces et territoires ont utilisé diverses stratégies pour encourager les parents à faire administrer tous les vaccins recommandés à leurs enfants. La définition, la portée, la souplesse (comme les exemptions pour des raisons médicales, religieuses et philosophiques) et les cadres légaux (comme la rigueur de l'application et le pouvoir d'application du mandat) varient considérablement d'un endroit à l'autre. Étonnamment, aucune différence marquée n'a cependant été observée dans les taux de vaccination des pays qui recommandent la vaccination et ceux qui la rendent obligatoire. Des conséquences imprévues des programmes d'immunisation obligatoire, tant positives (plus grande disponibilité des données) que négatives (contournement du système et répercussions disproportionnées sur les familles ayant un statut socioéconomique plus faible), ont été observées. S'attaquer au problème des faibles taux de vaccination est donc un problème complexe qui exige une approche en plusieurs volets, plus nuancée et mieux adaptée.
Cette oeuvre est mise à la disposition selon les termes de la licence internationale Creative Commons Attribution 4.0

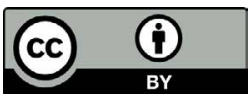

Affiliations

${ }^{1}$ Département de pédiatrie, Université Dalhousie, IWK Health Centre, Halifax, NS

2 Institut national de la santé publique du Québec, Département d'anthropologie, Université Laval, Québec, QC

${ }^{3}$ Département de médecine interne, Klinikum Saarbruecken, Allemagne

\section{${ }^{\star}$ Correspondance :}

noni.macdonald@dal.ca

Citation proposée : MacDonald NE, Dubé E, Grandt D. Éléments dont il faut tenir compte concernant les programmes d'immunisation obligatoire des enfants. Relevé des maladies transmissibles au Canada 2020;46(7/8):277-82. https://doi.org/10.14745/ccdr.v46i78a06f

Mots-clés : immunisation des enfants, obligatoire, CANVax

\section{Introduction}

Les éclosions de maladies évitables par la vaccination se produisent même dans les pays dont les revenus individuels sont élevés et qui ont un accès facile et relativement équitable à la vaccination. Elles s'expliquent notamment par le fait que les taux de vaccination ne sont pas aussi élevés qu'ils devraient l'être pour pouvoir s'assurer de contrôler efficacement les maladies pouvant être prévenues par la vaccination. Dans de nombreux pays, y compris au Canada, les parents hésitent à faire vacciner leurs nourrissons et leurs enfants à temps et dans les délais prescrits (1). C'est pour cette raison que plusieurs pays ont discuté d'une législation sur la vaccination obligatoire des enfants, avant d'adopter une telle législation ou de la renforcer, pour ainsi pouvoir s'attaquer au problème de réticence à la vaccination (2-4). La vaccination obligatoire est vue comme une solution " simple » au problème. L'inefficacité des stratégies et des mesures incitatives visant à atteindre les taux de vaccination souhaités, la réponse à une éclosion de maladie évitable par la vaccination qui est difficile à contrôler en raison du taux de vaccination plus bas que les taux souhaités et la pression pour atteindre un objectif d'élimination des maladies pouvant être prévenues par la vaccination, comme la poliomyélite (5).

Puisque des questions ont également été soulevées dans les médias afin de savoir si le ou les vaccins contre la maladie à coronavirus de 2019 (COVID-19), lorsqu'ils seront disponibles, devraient être rendus obligatoires pour certaines personnes seulement ou pour l'ensemble des Canadiens, ce dossier du Centre canadien de ressources et d'échange sur les données probantes en vaccination (CANVax) vise d'abord à donner un aperçu de ce que signifie l'immunisation obligatoire des enfants, puis comprend une discussion sur la portée et les cadres légaux dont il faut tenir compte. Il y est également question des 
résultats rapportés, incluant les rapports sur les conséquences imprévues.

Cet article est le sixième d'une série produite par CANVax, une base de données en ligne qui appuie la planification et la mise en œuvre des programmes de vaccination. Cette série d'articles inclut tant l'identification des ressources existantes que la description des nouvelles ressources développées par un groupe multidisciplinaire de professionnels (6). Cet article d'une série montre donc comment les divers aspects de la réticence à la vaccination examinés jusqu'à maintenant peuvent être utilisés pour favoriser l'acceptation des vaccins.

\section{Définition, portée et cadres des programmes d'immunisation obligatoire}

En 2010, un groupe d'experts a proposé une définition de vaccin " obligatoire " comme un vaccin que chaque enfant d'un pays, d'un état, d'une province ou d'un territoire doit recevoir en vertu de la loi sans que le parent ait la possibilité de l'accepter ou de le refuser, indépendamment de l'existence d'une conséquence ou d'une sanction ou d'une pénalité juridique ou économique en cas de refus (7). Les programmes de vaccination obligatoire varient énormément. II n'existe aucune approche uniforme pour la création de programmes de vaccination obligatoires ni de portée commune pour de tels programmes. Il est donc essentiel, lorsqu'on discute d'un programme obligatoire de vaccination des enfants (et des autres groupes d'âge), de comprendre ce que ce programme comporte et ce qu'on espérait qu'il permettrait de réaliser.

En ce qui concerne la vaccination des enfants, le mandat pourra s'appliquer tant à l'ensemble du pays (Italie (3) et la France (3)) ou à certains états, territoires ou provinces (la Californie, aux États-Unis (3) et l'Ontario, au Canada (8)). Il pourra cependant s'appliquer de façon plus spécifique à un sous-ensemble défini de la population des enfants (9). Ainsi, certains programmes couvrent la plupart des vaccins pour enfants recommandés par l'Organisation mondiale de la Santé (OMS) (e.g. I'Italie (3)) alors que d'autres visent plutôt une gamme limitée de vaccins (e.g. la France, qui couvre une liste précise (3)) et que d'autres, enfin, n'incluent qu'un seul vaccin (la Belgique, vaccin contre la poliomyélite (10)). Certains programmes peuvent aussi préciser un groupe d'âge ou un jalon important comme l'inscription à l'école (en maternelle en Italie) (3) et l'entrée à l'école en Californie, aux États-Unis. Sur le plan de la souplesse, certains programmes comportent des exemptions uniquement pour les contre-indications médicales, alors que d'autres incluent ou ont déjà inclus différentes exemptions pour des raisons religieuses et philosophiques (la Californie, aux États-Unis (4) et l'Australie (11) avant 2016).
Les cadres légaux, comme la rigueur de l'application et le pouvoir d'application du mandat, varient également, tout comme l'organisme responsable de l'application des exigences obligatoires (la Californie, aux États-Unis) (4). D'autres programmes peuvent, quant à eux, ne pas appliquer du tout le mandat (la Serbie). Ainsi, un programme peut mettre l'accent sur des mesures incitatives financières pour encourager la conformité (11) ou imposer des pénalités et des sanctions financières ou sociales (i.e. que les enfants peuvent être exclus des garderies (I'Ontario, au Canada (12) et l'Australie (11)) ou ne pas pouvoir entrer à l'école (la Californie, aux États-Unis). Les personnes peuvent également se voir interdire l'accès aux parcs thématiques (la Californie (12)) ou se voir imposer une amende (la Slovénie) (10) ou même une peine d'emprisonnement (l'Ouganda (13)).

Il existe donc une vaste gamme d'approches en ce qui concerne la vaccination obligatoire des enfants requise par la loi :

- Aucune mise en application, chacun peut choisir de ne pas se faire vacciner sans sanction ni pénalité (e.g. en France avant les changements mis en œuvre en 2018 (2))

- Retrait en raison d'une objection personnelle ou philosophique ne comportant ni sanction ni pénalité (e.g. en Ontario, avant les changements mis en œuvre en 2016 (14))

- Lois exigeant que les parents suivent une formation sur I'immunisation (plutôt que de recevoir la vaccination); retrait en raison d'une objection personnelle ou philosophique qui oblige cependant les parents à remplir des formulaires particuliers qui doivent ensuite être notariés, mais sans aucune sanction ni pénalité en cas de non-conformité (e.g. Ontario (8))

- Lois qui exigent la vaccination, mais auxquelles les personnes peuvent se soustraire si elles ont une objection personnelle ou philosophique. Ces lois exigent également de remplir des formulaires particuliers et demanderont des efforts supplémentaires. Une sanction ou pénalité sera cependant imposée pour toute non-conformité et cette loi est mise en application de façon stricte (e.g. l'Australie avant les changements en 2016 (11))

- Lois exigeant l'immunisation assorties de sanctions financières ou de restrictions sociales sévères. Seules les exemptions médicales sont autorisées et la mise en application de la loi est stricte (e.g. la Californie, aux États-Unis après $2016(4,15)$ et l'Australie après 2016 (11))

\section{Résultats des programmes de vaccination obligatoire}

Il existe étonnamment peu d'études systématiques et donc très peu de données comparatives sur les résultats des programmes de vaccination obligatoire des nourrissons et des enfants. Un rapport de 2006 n'a relevé aucune différence marquée dans les taux de vaccination entre les pays qui recommandent seulement 
certains vaccins et ceux qui les exigent (16). Ainsi, un examen systématique des résultats de ces législations effectué en 2016 n'a trouvé que 11 études avant et après, et seulement 10 études qui comparaient les taux d'immunisation dans des populations semblables avec et sans législation. Les auteurs ont donc conclu que dans l'ensemble, la vaccination obligatoire était généralement utile pour augmenter les taux de vaccination, bien que 18 des études incluses provenaient d'un seul pays, soit des États-Unis, et que deux autres provenaient du Canada et une de la France (17). Cet examen n'a pas permis d'évaluer l'incidence de la vaccination obligatoire sur les attitudes à l'égard de la vaccination.

En 2018, une étude de l'environnement législatif de la vaccination des enfants a été effectuée dans 53 pays de la région européenne (18). Les conclusions de cette étude ont montré une diversité de cadres législatifs en ce qui concerne la vaccination (des recommandations aux politiques obligatoires rigoureuses). Elle n'a cependant pas permis de déceler des données probantes claires sur la « meilleure approche " pour augmenter l'adoption et l'acceptation de la vaccination (i.e. qu'il n'y avait aucune corrélation entre les taux d'adoption et la présence ou l'absence de législation ou le type de législation). Pour interpréter correctement les résultats, il faut donc comprendre les différences entre les programmes de vaccination obligatoire dans un contexte historique et géographique. Les 15 républiques ethniques qui formaient I'Union des républiques socialistes soviétiques et ses voisins communistes avaient toutes des systèmes de santé publique centralisés très solides avec vaccination obligatoire qui permettaient d'appliquer la loi sans poser de questions et étaient donc assortis de taux de participation élevés. En 2018 toutefois, la situation n'était plus la même en ce qui concerne la vaccination des enfants dans bon nombre de ces pays. Ainsi, cette année-là, I'Ukraine a affiché le taux de vaccination infantile le plus faible de la région européenne établie par l'OMS, alors que la Serbie et la Pologne avaient vu des manifestations contre la vaccination obligatoire.

II n'y a cependant pas eu d'étude sur les mandats dans les administrations des pays à revenu élevé où les taux de vaccination de base sont relativement élevés ou dont le mandat inclut les garderies. En Belgique et en Italie, par exemple, certains vaccins étaient obligatoires pour des raisons historiques alors que d'autres ne l'étaient pas. Les vaccins non obligatoires peuvent donc avoir été vus par le public et les professionnels de la santé comme moins importants et moins nécessaires. En Italie, cet écart dans le programme de vaccination a permis d'obtenir une couverture vaccinale élevée (plus de $93 \%$ dans tous les cas) pour les vaccins obligatoires (e.g. diphtérie, tétanos, poliomyélite et hépatite $B$ ), mais les résultats ont grandement différé pour $d$ 'autres vaccins recommandés, mais non obligatoires puisque le taux de vaccination pour ces vaccins était inférieur à la couverture vaccinale nécessaire (e.g. la couverture de la rougeole était de 87 \%) (3). Les éclosions de rougeole ont donc poussé I'Italie à passer à une vaccination obligatoire plus étendue (3).
En Australie, en 2015, le projet de loi modificatif No Jab No Pay a supprimé, en raison des préoccupations concernant les taux de vaccination, l'exemption pour « objection de conscience " associée à la vaccination des exigences en matière de vaccination (11). Ainsi, en mars 2017, on a pu voir que ces changements s'étaient traduits par une augmentation de la vaccination chez les enfants de cinq ans, qui sont passés de $92,59 \%$ à $94,34 \%$ (10). Comme nous I'indiquons ci-dessous cependant, les répercussions de ce changement ont varié entre les différentes classes socioéconomiques.

En Ontario, le resserrement du processus obligatoire requis pour obtenir une exemption philosophique a permis de révéler des renseignements précieux, puisque les nouvelles données tirées de ces dossiers ont permis d'effectuer une analyse plus détaillée (19). Ainsi, en 2016-2017, 2,4 \% des élèves avaient une exemption non médicale pour au moins un antigène. On trouvait également d'autres élèves qui n'avaient pas encore été vaccinés, mais qui n'avaient demandé aucune exemption. En outre, le fait d'avoir une exemption non médicale signée n'était pas toujours lié à la non-immunisation. La probabilité d'avoir obtenu une exemption non médicale et de ne pas être vacciné était plus élevée dans les écoles privées ou dans d'autres écoles non financées par le gouvernement, ainsi que dans certaines régions géographiques particulières. De plus, les élèves plus âgés ou défavorisés étaient moins susceptibles d'avoir une exemption non médicale

\section{Conséquences imprévues des programmes de vaccination obligatoire}

Les programmes de vaccination obligatoire peuvent cependant avoir des répercussions imprévues. Ainsi, la suppression des exemptions non médicales (i.e. les exemptions pour convictions personnelles) a entraîné une augmentation des exemptions médicales en Californie, aux États-Unis (20) et en Australie (11). Les régions dans lesquelles les taux d'exemptions personnelles étaient élevés avant l'adoption des lois plus restrictives semblent avoir favorisé des taux plus élevés d'exemptions médicales. Cela suggère donc un " contournement » du système. Il est décevant de constater que la réponse de la population cible a été de demander une exemption médicale plutôt que d'accepter la vaccination.

En Australie, le programme d'immunisation obligatoire des enfants No Jab No Pay a augmenté le nombre de vaccinations comme indiqué ci-dessus, mais les enfants et les familles qui vivent dans la pauvreté ont été touchés de façon disproportionnée, ce qui a eu pour effet de susciter des préoccupations en matière d'équité et de justice (11).

Un avantage inattendu des programmes de vaccination obligatoire a porté sur la nécessité d'accorder une plus grande attention à la collecte de données sur les personnes vaccinées. Ce fut notamment le cas en Ontario où l'on a consacré du temps, 
de l'attention et des fonds pour assurer le fonctionnement du registre de vaccination des enfants.

\section{Vaccins contre la COVID-19 et examen d'une approche obligatoire}

Bien qu'un sondage effectué au Canada à la fin d'avril 2020 ait révélé que le public était majoritairement en faveur de rendre la vaccination obligatoire pour la COVID-19 (21), cette stratégie ne pourra être envisagée que lorsque ces vaccins deviendront facilement disponibles et accessibles au Canada. Puisqu'un programme de vaccination obligatoire comporte des coûts tant sur le plan de la mise en œuvre que de la surveillance (5), les décisions doivent reposer sur les avantages supplémentaires que l'on espère obtenir. Si l'on s'attend déjà à ce que le taux de vaccination soit élevé parmi les groupes jugés nécessaires pour contrôler la propagation de la COVID-19, les coûts supplémentaires d'un programme obligatoire ne seraient probablement pas justifiés. En revanche, si les taux de participation sont faibles et que la facilité d'accès et d'autres stratégies connues visant à augmenter la participation ont été prises en compte, une approche obligatoire pourrait alors être appropriée. Il faut donc porter une attention particulière à la question de savoir s'il s'agira d'un programme avec mesures incitatives, sanctions ou pénalités, qui en effectuera la surveillance et de quelle façon il sera surveillé (5).

\section{Conclusion}

II n'existe aucune approche globale normalisée en ce qui concerne la vaccination obligatoire. II faut donc déterminer quels vaccins seront inclus, quels groupes d'âge seront couverts, ainsi que le niveau de souplesse et de rigidité du programme (e.g. possibilités de retrait, pénalités, sanctions ou mesures incitatives et degré d'application de la loi). La vaccination obligatoire des enfants ne garantit pas que le problème des taux de vaccination inférieurs à ceux souhaités sera résolu, bien qu'il puisse entraîner une augmentation de la vaccination. II n'y avait aucune différence marquée dans les taux de vaccination entre les pays qui recommandent seulement certains vaccins et les pays qui les exigent. Le contexte joue également un rôle important puisqu'il permet à un pays de décider s'il doit ou non mettre en œuvre la vaccination obligatoire en raison des raisons et des circonstances différentes et de choisir la ou les méthodes à utiliser pour ce faire. Il faut également prévoir les conséquences imprévues d'un tel programme comme un taux réduit d'acceptation des vaccins non obligatoires, ainsi que la possibilité que des personnes qui hésitent à se faire vacciner contournent le système. Des exigences rigides en matière de vaccination obligatoire semblent, à première vue, la solution simple pour améliorer les taux de vaccination. Les données probantes n'étayent cependant pas fortement cette conclusion. La vaccination obligatoire $n^{\prime}$ est qu'une des stratégies à envisager. S'attaquer au problème du faible taux de vaccination est un problème complexe qui nécessite une approche en plusieurs volets, plus nuancée et adaptée (22).

\section{Déclaration des auteurs}

N. E. M. - Rédaction du projet original

E. D. - Rédaction, révision et correction

D. G. - Rédaction, révision et correction

\section{Intérêts concurrents}

La Dre Macdonald a reçu des subventions de l'Agence de la santé publique du Canada, des Instituts de recherche en santé du Canada, de la Régie de la santé de la Nouvelle-Écosse, de la IWK Health Authority et du Réseau canadien de recherche sur I'immunisation. La D're Dubé a reçu des subventions de l'Agence de la santé publique du Canada, du ministère de la Santé et des Services sociaux du Québec, du Fonds de la recherche en Santé du Québec, des Instituts de recherche en santé du Canada, du Réseau canadien de recherche sur l'immunisation et du Conseil de recherches en sciences humaines du Canada.

\section{Remerciements}

Les contributions au CANVax proviennent d'un vaste éventail d'auteurs, de comités, de partenaires en immunisation, d'examinateurs et surtout du secrétariat du CANVax de l'Association canadienne de santé publique.

\section{Références}

1. Lane S, MacDonald NE, Marti M, Dumolard L. Vaccine hesitancy around the globe: analysis of three years of WHO/ UNICEF Joint Reporting Form data-2015-2017. Vaccine 2018;36(26):3861-7. DOl PubMed

2. Ward JK, Colgrove J, Verger P. Why France is making eight new vaccines mandatory. Vaccine 2018;36(14):1801-3. DOI PubMed

3. Ricciardi W, Boccia S, Siliquini R. Moving towards compulsory vaccination: the Italian experience. Eur J Public Health 2018;28(1):2-3. DOI PubMed

4. Delamater PL, Leslie TF, Yang YT. Change in medical exemptions from immunization in California after elimination of personal belief exemptions. JAMA 2017;318(9):863-4. DOI PubMed

5. MacDonald NE, Harmon S, Dube E, Steenbeek A, Crowcroft N, Opel DJ, Faour D, Leask J, Butler R. Mandatory infant \& childhood immunization: Rationales, issues and knowledge gaps. Vaccine 2018;36(39):5811-8. DOI PubMed

6. Association canadienne de santé publique. Centre canadien de ressources et d'échange sur les données probantes en vaccination. Ottawa (ON) : CANVax (accédé 2019-12-15). https://www.canvax.ca/fr 
7. Haverkate M, D'Ancona F, Giambi C, Johansen K, Lopalco PL, Cozza V, Appelgren E; VENICE project gatekeepers and contact points. Mandatory and recommended vaccination in the EU, Iceland and Norway: results of the VENICE 2010 survey on the ways of implementing national vaccination programmes. Euro Surveill 2012;17(22):20183. DOI PubMed

8. Dyer O. Ontario suspends unvaccinated children from school and proposes mandatory classes for parents. BMJ 2015;351:h6821. DOI PubMed

9. Yezli S. The threat of meningococcal disease during the Hajj and Umrah mass gatherings: A comprehensive review. Travel Med Infect Dis Jul - Aug 2018;24:51-8. DOI PubMed

10. Walkinshaw E. Mandatory vaccinations: the international landscape. CMAJ 2011 Nov;183(16):E1167-8. DOI PubMed

11. Leask J, Danchin M. Imposing penalties for vaccine rejection requires strong scrutiny. J Paediatr Child Health 2017 May;53(5):439-44. DOl PubMed

12. Government of Ontario. Child Care and Early Years Act, 2014, S.O. 2014, c. 11, Sched. 1. Immunization. https://www.ontario.ca/laws/regulation/150137\#BK46

13. Uganda Legal Information Institute. Immunisation Act, 2017. https://ulii.org/node/27644

14. Wilson SE, Seo CY, Lim GH, Fediurek J, Crowcroft NS, Deeks SL. Trends in medical and nonmedical immunization exemptions to measles-containing vaccine in Ontario: an annual cross-sectional assessment of students from school years 2002/03 to 2012/13. CMAJ Open 2015;3(3):E317-23. DOI PubMed

15. Zipprich J, Winter K, Hacker J, Xia D, Watt J, Harriman K; Centers for Disease Control and Prevention (CDC). Measles outbreak--California, December 2014-February 2015. MMWR Morb Mortal Wkly Rep 2015;64(6):153-4. PubMed
16. Salmon DA, Teret SP, Maclntyre CR, Salisbury D, Burgess MA, Halsey NA. Compulsory vaccination and conscientious or philosophical exemptions: past, present, and future. Lancet 2006;367(9508):436-42. DOI PubMed

17. Lee C, Robinson JL. Systematic review of the effect of immunization mandates on uptake of routine childhood immunizations. J Infect 2016;72(6):659-66. DOI PubMed

18. Sabin Vaccine Institute. Legislative Landscape Review: Legislative Approaches to Immunization Across the European Region. Washington (DC): Sabin Institute; 2018. https://www.sabin.org/sites/sabin.org/files/legislative_ approaches_to_immunization_europe_sabin_0.pdf

19. Wilson SE, Murray J, Bunko A, Johnson S, Buchan SA, Crowcroft NS, Dubey V, Loh LC, MacLeod M, Taylor C, Deeks SL. Characteristics of immunized and un-immunized students, including non-medical exemptions, in Ontario, Canada: 2016-2017 school year. Vaccine 2019;37(23):312332. DOI PubMed

20. Delamater PL, Pingali SC, Buttenheim AM, Salmon DA, Klein NP, Omer SB. Elimination of nonmedical immunization exemptions in California and school-entry vaccine status. Pediatrics 2019;143(6):e20183301. DOI PubMed

21. Leger. Concerns about COVID-19 - April 28, 2020. https://leger360.com/surveys/concerns-about-covid-19april-28-2020/

22. Dubé E, Bettinger JA, Fisher WA, Naus M, Mahmud SM, Hilderman T. Acceptation, refus et hésitation à la vaccination au Canada : défis et approches proposées. Relevé des maladies transmissibles au Canada 2016;42(12):274-9. DOI 


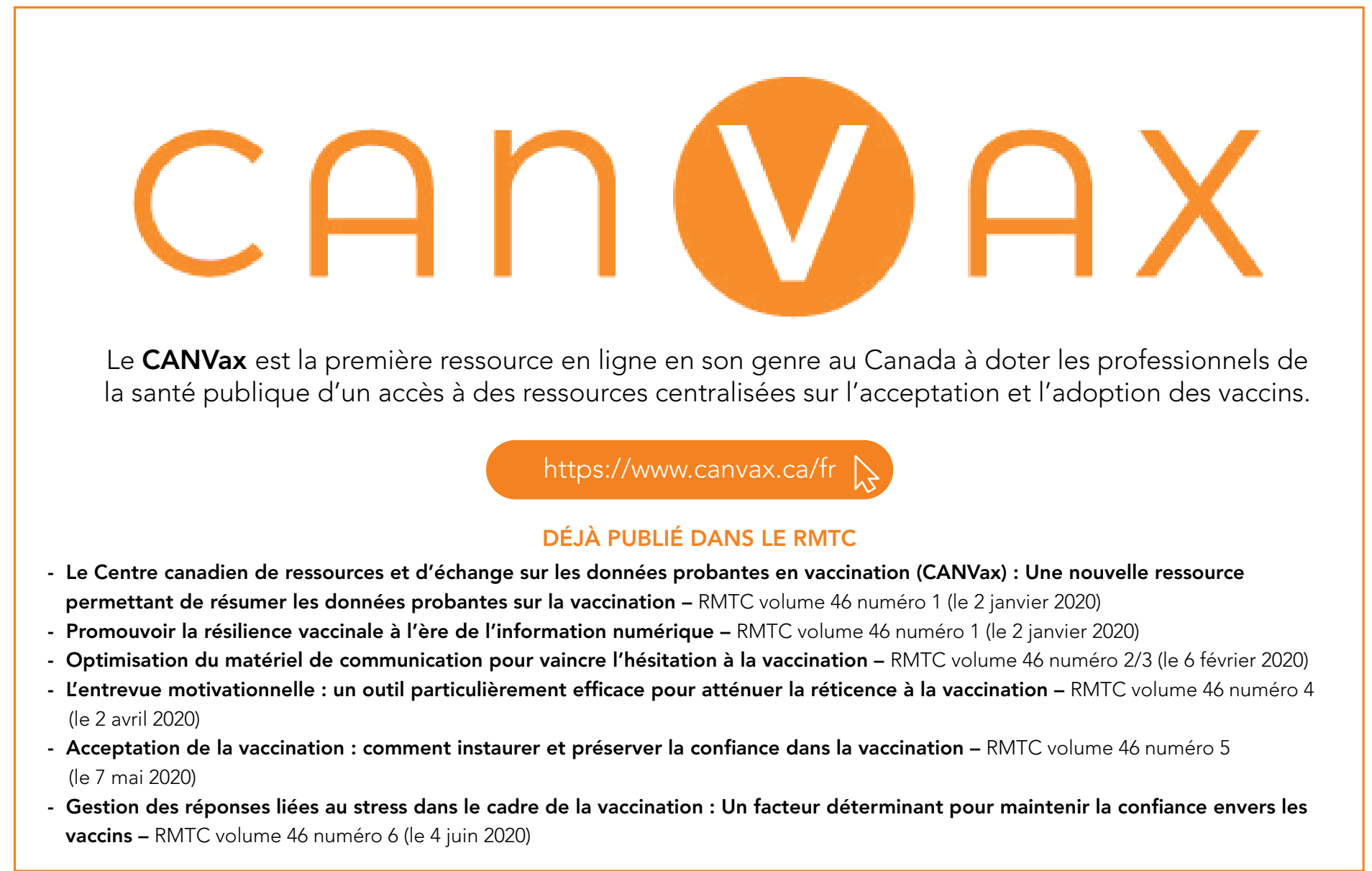

